\title{
An Evaluation of Clinical Spectrum of Leprosy in and Around a Tertiary Care Hospital in North India
}

\author{
Dr. Deepti Gangwar ${ }^{1}$,Dr. Vivek Gupta ${ }^{2}$,Dr. Sunil Gupta ${ }^{3}$,Dr. D.K. Jain ${ }^{4}$,Dr \\ Shashwat Vidyadhar \\ ${ }^{I}$ Resident Department Of Pathology, Hind Institute Of Medical Sciences, Barabanki, U.P. \\ ${ }^{2}$ M.D.(Pathology) Professor \& Head, Department Of Pathology, Hind Institute Of Medical \\ Sciences, Barabanki, U.P. \\ ${ }^{3}$ M.D., Associate Prof, Department Of Skin And V.D. Hind Institute Of Medical Sciences, Barabanki, U. P. \\ ${ }^{4}$ M.D., Assistant Prof. Department Of Pathology, Hind Institute Of Medical Sciences, Barabanki, U. P. \\ ${ }^{5}$ Resident Department Of Pathology, Hind Institute Of Medical Sciences, Barabanki, U.P
}

\begin{abstract}
:
Background:-Leprosy(Hansen's disease)is a chronic infectious disease caused by Mycobacterium leprae. It is classified into 5 groups based on clinical,bacteriological,immunological and histopathological status of patients(Ridley -Jopling classification).However a great variation has been observed in the interpretation of histopathological examination of skin biopsies and clinical presentation of disease

Objectives:-To correlate clinical diagnosis with histopathological diagnosis of leprosy patients in

Tertiary care hospital in north India

Material and Methods:-The presents study includes all the new leprosy patients visiting the OPD of skin and V.D. department, HIMS, Safedabad, Barabanki,U.P.It is a hospital based cross-sectional study conducted among patients with all clinical types of leprosy,classified as per Ridley-Jopling classification. Skin biopsies were taken from active lesion in all patients and were stained with $H$ and $E$ stain for identification of Mycobacterium leprae ,slit smears were done to study bacillary index.All clinicopathologically diagnosed cases of TTHD and BTHD will be subjected to $S-100$ stain.

Results: More than one third of the patients were between 31-40 years (39.6\%) followed by 20-30 (18.9\%), 41$50(17 \%),>50(13.2 \%)$ and $<20(11.3 \%)$. More than half of the patients were males $(11.3 \%)$. The family history of leprosy was present among $9.4 \%$ of the patients. Hypopigmented skin lesion was the most common clinical symptom (32.1\%). TT was the most common clinical diagnosis (26.4\%). IL was the most common histopathological diagnosis (26.4\%). Atrophy epidermis changes was found in $50.9 \%$ patients. Pauci bacillary was found in $62.3 \%$ of the patients.

Conclusion: Leprosy though reported to be eliminated still continues to be one of the common infectious diseases in Uttar Pradesh, India and necessitates revival of our knowledge regarding the disease. Skin biopsies, slit smears and IHC S-100 are useful tools in confirming the clinical diagnosis of leprosy as well as for the therapeutic guide.
\end{abstract}

Keywords: Clinical spectrum, Leprosy, Histopathology

\section{Introduction}

Leprosy also known as Hansen's disease and is the oldest disease known to mankind. In India, leprosy was first described in susruth samhita written in $600 \mathrm{BC}{ }^{(1)}$. Leprosy is known, since ancient times as "Kushtaroga", whose clinical manifestations are largely confined to the skin, peripheral nervous system, upper respiratory tract, eyes and testes. The three cardinal sign of the disease are skin lesions, skin anesthesia and enlarged peripheral nerves Although in January 2006 leprosy was eliminated in India but it is still a public health problem in the country ${ }^{(3)}$ In India, a total of 0.92 lac cases were on record as on April 2013, giving a Prevalence rate (PR) of 0.73 per 10,000 population and in Rajasthan 0.12 lac cases were on record as on April 2013 , giving a prevalence rate of 0.18 per 10,000 population Leprosy is one of the leading causes of physical disabilities contributing to intense social stigma resulting in human discrimination. This chronic infectious disease caused by Mycobacterium Leprae principally affects skin and peripheral nerves; it also involves muscles, eyes, bones, testis and internal organs. The clinical manifestations are varied ranging from an insignificant skin lesion to extensive disease causing profound disability/deformities ${ }^{(5)}$

The objective of this study was to evaluate the clinical spectrum of leprosy in and around a tertiary care hospital in north India. 


\section{Material And Methods}

This study was a hospital based cross-sectional study. The study included all the new leprosy patients visiting the OPD of Skin and V.D, HIMS, Safedabad, Barabanki. UP. The study was performed after permission from the Institutional Ethical Committee. The study duration was one year. All skin lesions like hypo pigmented patch, anesthetic patch, pure nerve thickening attending skin \& V.D leprosy OPD were included in the study. Patients with partially treated cases, immunological parameters in Ridley - Jopling classification were excluded from the study.

\section{Methods}

S-100 immonoperoxidase staining - All clinicohistopathologically diagnosed cases of TTHD \& BTHD will be subjected to S- 100 stain (Dako). Clinical diagnosis, histopathological features and S-100 staining patterns was then be correlated. Patients were clinically evaluated in a well illuminated room for any skin lesion, nerve thickening etc. Mandatory punch biopsy skin and wherever possible neural FNAC preferably from sural nerve was done. The tissue was sent to histopatholgy section of department of pathology for tissue processing, sections cutting \& routine H\&E staining, Fite - Faracco staining for detecting lepra bacilli (in slit smears)

\section{Modified Z-N stain or Fite -Faracco staining for lepra bacilli}

1. Slide with the smear should be covered with carbol fuchin and heat applied beneath it,either with gas flame (Bunsen burner)or with a spirit lamp.Heating should be sufficient to cause steam to rise from all parts of the slide,but boiling is avoided.The slide should be left for 15 minutes without any further heating; 2 . The stain is tipped away and the slide is held under a gentle stream of water; 3 . Pour acid-alcohol mixture on to the slide and leave for 3 seconds if the smear is thick,then wash it away with running water.The acid- alcohol mixture consist of $1 \%$ hydrochloric acid in $70 \%$ alcohol .The slide is inspected to see the degree of pinkness; if faintly pink proceed to next stage,but deeply pink treat again with acid-alcohol for 2 seconds and wash with running water; 4 . Cover the slide with counter -stain such as $1 \%$ methylene blue for about 10 seconds; and 5. Washed in running water and allow to dry)

\section{Bacterial indices:-}

6+ Many clumps of bacilli in an average field (over 1000), 5+ 100-1000 bacilli in average field, 4+ 10100 bacilli in an average field, 3+1-10 bacilli in an average field, 2+ 1-10 bacilli in 10 fields and 1+1-10 bacilli in 100 fields.

\section{Statistical analysis}

The descriptive statistics are presented. The Chi-square test was used for comparisons. The p-value $<0.05$ was considered significant. All the analysis was carried out on SPSS 16.0 version (Chicago, Inc., USA).

The study of pathological changes in leprosy lesions has contributed a great deal to understanding of the disease and clinico-pathological correlative studies have provided further insights into the disease, its varied manifestations and complications. Pathological examination helps to confirm a presumptive clinical diagnosis and also helps for exact typing.

\section{The following are the salient findings of this study:}

The study was conducted on 53 cases of leprosy in and around Safedabad,Barabanki. Majority of patients with leprosy were between 31-40 years of age (39.6\%) followed by 20-30 years(18.9\%), 41-50years $(17 \%)$, greater than 50 years (13.2\%) and less than 20years (11.3\%) (Table-1) Leprosy was most common among males $34(64.2 \%)$ and in females it was $19(35.8 \%)$. Family history of leprosy was present in $(9.4 \%)$ of cases

\section{Clinical Features ( In 53 Cases):-}

Most common clinical symptom was hypopigmented skin lesion (32.1\%) followed by anaesthesia (loss of sensation)(18.9\%), nerve thickening (17\%) \& erythematous skin lesions(17\%), combinations of lesions (7.5\%) and nodules(3.8\%) \& least common was trophic ulcers (3.8\%).(Table-2) According to Ridly-Jopling histopathological classification TT was most common clinical diagnosis (26.6\%), rest were as follows in descending order $\mathrm{LL}(22.6 \%), \mathrm{BT}(17 \%), \mathrm{IL}(13.2 \%), \mathrm{BB}(13.3 \%), \mathrm{BL}(9.4 \%)$.

Most common duration of leprosy was 12-24 months in majority of the patients (66\%) followed by 6-11 months (20.8\%), less than 6 months( $(9.4 \%)$ and least common duration was more than 24 months (3.8\%).

TT (26.4\%) was the most common clinical diagnosis followed by LL (22.6\%), BT (17\%), IL (13.2\%), and BB (11.3\%). Least common was BL (9.4\%). Clinical diagnosis with age. TT $3(42.9 \%)$ was most common in the age group more than 50 Years .BB $3(50 \%)$ was most common in the age 20 years. LL 4 (40\%) was most common in the age group of 20-30 years .BL $3(30 \%)$ was most common in age group 20-30 years . BT 2 
$(28.65 \%)$ was most common in age group greater than 50 years $(28.6 \%)$. IL $2(22.2 \%)$ was most common in age group 41-50 years.

\section{Histopathological Features (In 53 Cases):- Dermis:-}

Epithelioid Granuloma was the most common histological dermal change seen in $(20.8 \%)$ followed by giant cells seen in $(18.9 \%)$, perivascular lymphocytes were seen in $(17 \%)$, periappendageal lymphocytes seen in (15.1\%),perineural lymphocytes seen in(13.2\%), grenz zone seen in $(9.4 \%)$ and periappendageal lymphohistiocytes seen in (5.7\%).(Table-3) According to Ridly-Jopling histopathological classification IL was most common histopathological diagnosis $(26.4 \%)$ rest were as follows in descending order BT (22.6\%), LL(20.8\%), BB(13.2\%), TT(7.5\%), BL(9.4\%) Examination of slit smear showed paucibacillary (62.3\%), multibacillary20 (37.7\%),. 2+ type of bacillary index in multi bacillary was most common 6 (30\%).(Fig-4) On comparison of clinical diagnosis with histopathological diagnosis. BB was correlated in (16.7\%) BT was correlated in $(11.1 \%)$ of the cases, LL was correrated in $(8.3 \%)$. However, TT correlated in $(7.1 \%)$ of the cases. From this study it was concluded that immunostaining with S-100 Protein (Dako) facilitated detection of nerve elements within granuloma.Thus helping in making the diagnosis of tuberculoid (TT) and borderline tuberculoid(BT) cases

\section{Discussion}

Leprosy, also known as Hansen's disease, is a chronic, granulomatous, infectious disease that primarily affects the skin and the peripheral nerves. It is a spectral disease in which the clinical and pathological features reflect the cell-mediated immunity of the host, so it needs an appropriate classification because of its varied manifestations. The WHO classification ${ }^{(6)}$ of dividing leprosy into PB $(<5$ lesions) and MB ( $\geq 5$ lesions) is recommended for routine use and either Indian or Ridley-Jopling classification for research workers The study included 53 patients ranging from 12 to 60 years which showed slightly higher preponderance in males. This indicates their more vulnerability because of greater mobility and increased opportunities for contact in big population $^{(8)}$. Though most patients were middle aged (31-40), $11.3 \%$ were children and adolescents $<20$ years of age. This was comparable with the study by Thakkar and Patel $(2014)^{(9)}$ in which higher preponderance of leprosy cases was found in males and in middle aged.

Positive family/contact history $(9.4 \%)$ was comparable with the study of Salodkar and Kalla (1995) ${ }^{(10)}$ which showed it in $9.5 \%$ of the cases as well as the study by Thakkar and Patel (2014) $(8.3 \%) .{ }^{(9)}$

In the present study, hypopigmented skin lesions was the most common clinical symptom $(32.1 \%)$ followed by Anaesthesia (loss of sensation) (18.9\%), Nerve thickening \& Erythematous skin lesions (17\%), Combinations of lesions $(7.5 \%)$ and Nodules \& Trophic ulcers $(3.8 \%)$. Similar observations were made by Verma $(1976)^{(11)}$. Since skin and nerves are the commonest sites of M. leprae infection, signs and symptoms related to skin and nerves were common. In contrast, hypopigmented skin lesions were the most common clinical feature in the study by Murthy $(2000)^{(12)}$.

In the present study, the histopathological features of epidermis revealed that Atrophy epidermis changes was found in $50.9 \%$ patients. However, unremarkable was in $20.8 \%$ and ulceration was in $15.1 \%$. Basement membrane erosion was seen in $13.2 \%$ of the patients. The location of the granuloma mostly in relation to the deep and mid dermal nerves and neurovascular complexes would account for the somatosensory and autonomic neuropathic manifestations of the disease ${ }^{(13)}$.

Also the granuloma situated in mid dermis or in deep dermis and/or small granuloma, is unlikely to cause epidermal changes and hypopigmented skin lesions. When it reaches the superficial dermis and extends to the epidermis, atrophy of epidermis and development of hypopigmented lesions occur ${ }^{(13)}$.

In the present study, the Pauci bacillary was found in $62.3 \%$ of the patients which was similar to the findings of Suri et al (2014) ${ }^{(5)}$.

Clinical spectrum of leprosy cases in the present study revealed that TT was the most common clinical diagnosis $(26.4 \%)$ followed by IL (22.6\%), BT (17\%), IL (13.2\%), BB (11.3\%) and BL (9.4\%). Similar predominance of cases in the borderline group was also observed by Sharma et al $(2008)^{(14)}$, Shenoi and Sidappa $(1988)^{(15)}$, Nadkarni and Rege (1999) $)^{(16)}$ and Moorthyet al $(2001)^{(17)}$.

In this study, the histopathology finding revealed that IL was the most common histopathological diagnosis $(26.4 \%)$ followed by BT $(22.6 \%)$, LL (20.8\%), BB (13.2\%), TT (7.5\%) and BL (9.4\%). However, Thakkar and Patel $(2014)^{(9)}$ found that histopathology suggested the findings of BL. This highlights the value of slit skin smear as well as histopathology in suspected cases.

TT is slightly different from BT leprosy, both clinically and histopathologically. The line of demarcation often overlaps. Many cases diagnosed clinically as TT have histological evidence of BT ${ }^{(18)}$. TT is the infection on a knife edge, poised between progression and spontaneous healing. Though the borderline leprosy group is common and BT in particular is the commonest type of leprosy encountered in the world, many 
disagree with it. Failure to appreciate this fact is due to failure to recognize the exact clinical and histological features and the tendency to classify BT as $\mathrm{TT}^{(19)}$

Leprosy cases showed so many diversities between the clinical and histopathological features. In the present study, the histopathological characteristics were consistent with the clinical diagnosis in most of the patients. In a study conducted by Shenoi and Siddappa $(1988)^{(15)}$ and Singh et al $(1983)^{(21)}$, the histopathological features were consistent with clinical diagnosis in $47 \%$ and $47.6 \%$ respectively. The disparity was found in few cases, which is in concordance to the observations recorded by Shenoi and Siddappa (1988) ${ }^{(15)}$, Moorthyet al $(2001)^{(17)}$ and Nadkarni and Rege $(1999)^{(22)}$.

Clinical diagnosis of early leprosy lesions offer difficulties even to experienced dermatologists. A definitive diagnosis may be possible by histopathological examination. Histopathological features indicate the accurate response of the tissue, while the clinical features indicate only the gross morphology of the lesions which is due to underlying pathological changes.

\section{Conclusion}

Leprosy though reported to be eliminated still continues to be one of the common infectious diseases in Uttar Pradesh, India and necessitates revival of our knowledge regarding the disease. Skin biopsies, slit smears and IHC S-100 are useful tools in confirming the clinical diagnosis of leprosy as well as for the therapeutic guide.

\section{Conflict of interest}

None

Funding

None

\section{References}

[1]. Lowe J. Comments on the history of Leprosy. Lepr Rev 1947; 18: p. 54-63.8.

[2]. Hastings RC, Gillis TP, Krahenbuhl JL, Franzblau SG. Leprosy.ClinMicrobiol Rev 1988; 1: 330-348

[3]. Announcement: India achieves national elimination of leprosy. Indian J Lepr 2006; 78: 101.

[4]. NLEP - Progress Report for the year 2012-13, Central Leprosy Division Directorate General of Health Services Nirman Bhawan, New Delhi.

[5]. Suri Sushil kumar K, IyerRekha R, Patel Darshana U, BandilSupriya, Baxi Seema. Histopathology and Clinico-histopathological correlation in Hansen's disease.J Res Med Den Sci.2014; 2(1): 37-44

[6]. World Health Organizion. Chemotherapy of leprosy for control programmes. WHO Tech Rep Ser 675. Geneva: WHO; 1982.

[7]. Sachdeva S, Amin SS, Khan Z, Alam S, Sharma PK. Childhood leprosy: A retrospective study. J Public Health Epidemiol 2010;2:267-71.

[8]. Indian association of Leprologists- approved classification. Lepr India 1982;54:22-32

[9]. Thakkar S, Patel SV. Clinical profile of leprosy patients: A prospective study. Indian J Dermatol 2014;59:158-62

[10]. Salodkar AD, Kalla G. A clinicoepidemiological study of leprosy in arid North west Rajasthan, Jodhpur: Ind J Lepr 1995;57:161-6

[11]. Verma OP. Some epidemiological features of leprosy in a rural area in Hooghly District.Lepr India 1976; 48 (4): 371 -81.

[12]. Murthy N. Histopathological study of leprosy (unpublished Doctoral dissertation Rajiv Gandhi University of Health Sciences, 2000).

[13]. Suneetha S, Arundhathi S, Chandi S, Kurian N, Chacco CJG. Histological studies in primary neuritic leprosy: Changes in the apparently normal skin. Lepr Rev 1998; 69: 351-7.

[14]. Sharma A, Sharma RK, Goswsami KC, Bardwaj S. Clinico-Histopathological Correlation in Leprosy. JK Science 2008;10:120-3.

[15]. Shenoi SD, Siddappa K. Correlation of clinical and histopathologic features in untreated macular lesions of leprosy: A study of 100 cases. Ind J Lepr 1988;60:202-6.

[16]. Nadkarni NS, Rege VL. Significance of histopathological classification in leprosy.Ind J Lepr 1999;7:325-32.

[17]. Moorthy BN, Kumar P, Chatura KR, Chandrasekhar HR, Basavaraja PK. Histopathological correlation of skin biopsies in leprosy. Ind J DermatolVenLeprol 2001;67:299-301.

[18]. Bhatia AS, Katoch K, Narayanan RB, Ramu G, Mukherjee A, Lavania RK. Clinical and Histopathological correlation in the classification of leprosy.Int J Lepr 1993; 61 (3): 433-8.

[19]. Jopling WH, McDougall AC. The Disease. In: Handbook of Leprosy. 5th ed, Delhi: CBS Publishers and Distributors; 1996. p.1053.

[20]. Ridley DS, Jopling WH. A classification of leprosy for research purposes.Lepr Rev. 1962;33:119-128

[21]. Singh K, Iyengar B, Singh R. Variation in clinical and histopathological classification of leprosy: A report and plausible explanation. Lepr India 1983;55:472-9.

[22]. Nadkarni NS, Rege VL. Significance of histopathological classification in leprosy.Ind J Lepr 1999;7:325-32.

[23]. Singh K, Jyengar B, Singh R. Variations in Clinical and Histopathological classification of leprosy - a report and a plausible explanation. Lepr India 1983; 55 (3): 472-8.

Table-1: Distribution of patients according to demographic profile

\begin{tabular}{|l|c|c|}
\hline & No. $(\mathbf{n = 5 3})$ & \% \\
\hline Age in years & & 11.3 \\
\hline$<20$ & 6 & 18.9 \\
\hline $20-30$ & 10 & 39.6 \\
\hline
\end{tabular}


An Evaluation Of Clinical Spectrum Of Leprosy In And Around A Tertiary Care.....

\begin{tabular}{|l|c|c|}
\hline $41-50$ & 9 & 17.0 \\
\hline$>50$ & 7 & 13.2 \\
\hline Gender & & \\
\hline Male & 34 & 64.2 \\
\hline Female & 19 & 35.8 \\
\hline Family history & & \\
\hline Present & 5 & 9.4 \\
\hline Absent & 48 & 90.6 \\
\hline
\end{tabular}

Table-2: Distribution of patients according to clinical symptoms

\begin{tabular}{|l|c|c|}
\hline \multicolumn{1}{|c|}{ Clinical symptoms } & $\begin{array}{c}\text { No. } \\
(\mathbf{n = 5 3 )}\end{array}$ & \% \\
\hline Anaesthesia (loss of sensation) & 10 & 18.9 \\
\hline Hypopigmented skin lesions & 17 & 32.1 \\
\hline Nerve thickening & 9 & 17.0 \\
\hline Erythematous skin lesions & 9 & 17.0 \\
\hline Combinations of lesions & 4 & 7.5 \\
\hline Nodules & 2 & 3.8 \\
\hline Trophic ulcers & 2 & 3.8 \\
\hline
\end{tabular}

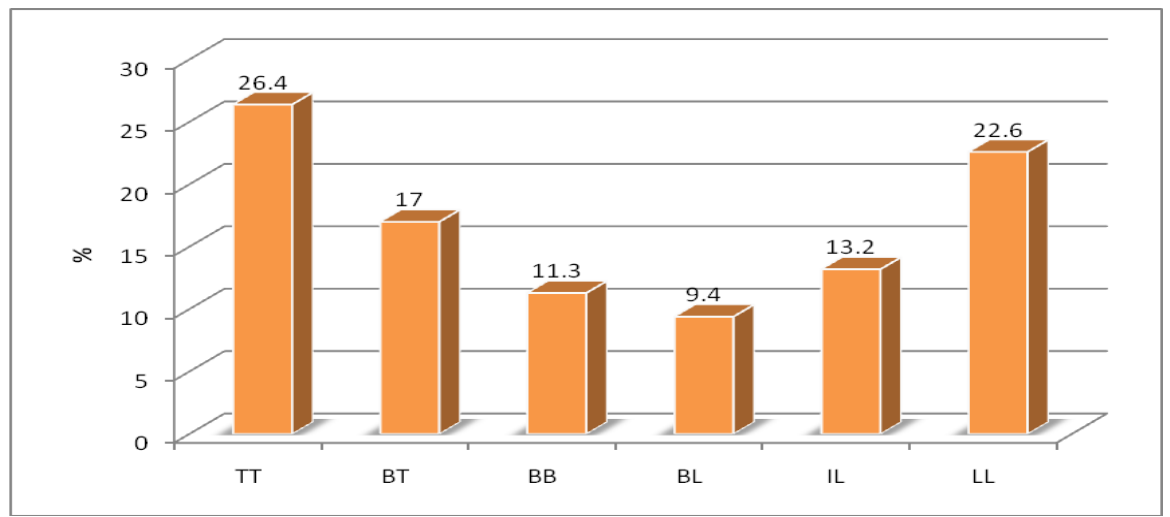

Fig. 1: Distribution of patients according to clinical diagnosis of leprosy

(TT: Tuberculoid leprosy, BT: Borderline tuberculoid, BB: Borderline borderline leprosy, BL: orderline lepromatous leprosy, IL: Indeterminate leprosy, LL: Lepromatous leprosy)

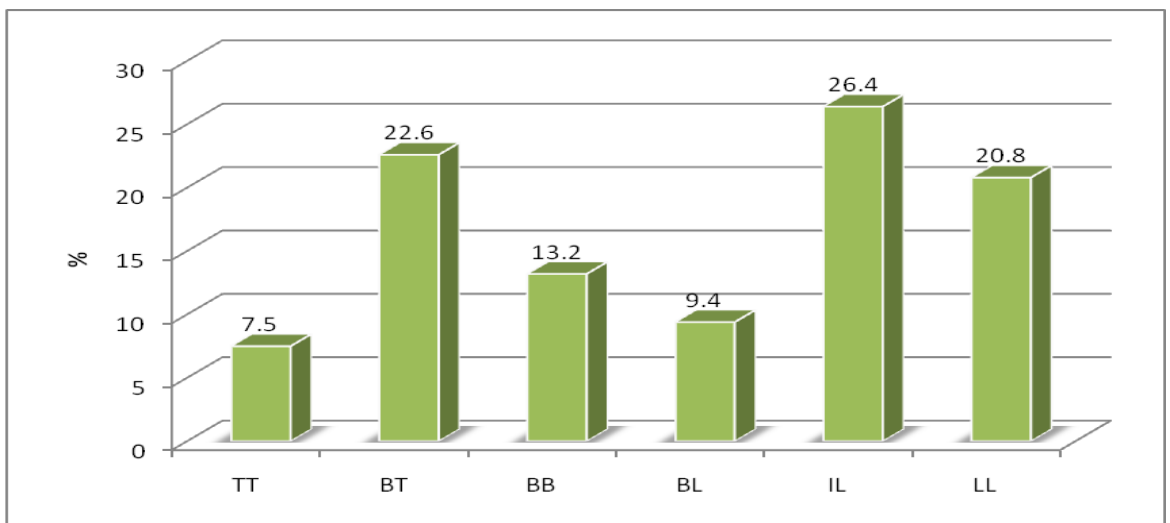

Fig. 2: Distribution of patients according to histopathological diagnosis of leprosy (TT: Tuberculoid leprosy, BT: Borderline tuberculoid, BB: Borderline borderline leprosy, BL: orderline lepromatous leprosy, IL: Indeterminate leprosy, LL: Lepromatous leprosy)

Table-3: Distribution of patients according to histological epidermis changes in leprosy

\begin{tabular}{|l|c|c|}
\hline \multicolumn{1}{|c|}{ Histopathological changes } & $\begin{array}{c}\text { No. } \\
(\mathbf{n = 5 3 )}\end{array}$ & \% \\
\hline Epidermis changes & & 50.9 \\
\hline Atrophy & 27 & 15.1 \\
\hline Ulceration & 8 & \\
\hline
\end{tabular}


An Evaluation Of Clinical Spectrum Of Leprosy In And Around A Tertiary Care.....

\begin{tabular}{|l|c|c|}
\hline Unremarkable & 11 & 20.8 \\
\hline Basement membrane erosion & 7 & 13.2 \\
\hline Dermal changes & & \\
\hline Epithelioid Granuloma & 11 & 20.8 \\
\hline Giant cells & 10 & 18.9 \\
\hline Periappendageal Lymphocytes & 8 & 15.1 \\
\hline Perivascular Lymphocytes & 9 & 17.0 \\
\hline Perineural Lymphocytes & 7 & 13.2 \\
\hline PeriappendagealLymphohistiocytes & 3 & 5.7 \\
\hline Grenz Zone & 5 & 9.4 \\
\hline
\end{tabular}

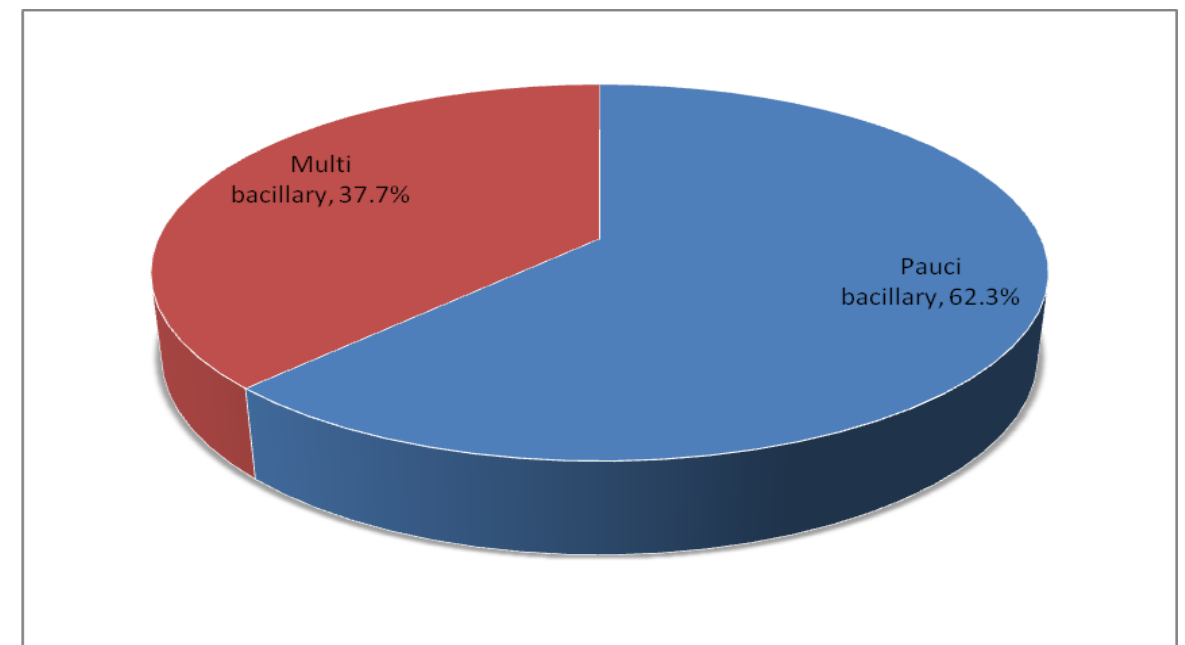

Fig. 3: Distribution of Bacillary Index in skin slit smear examination

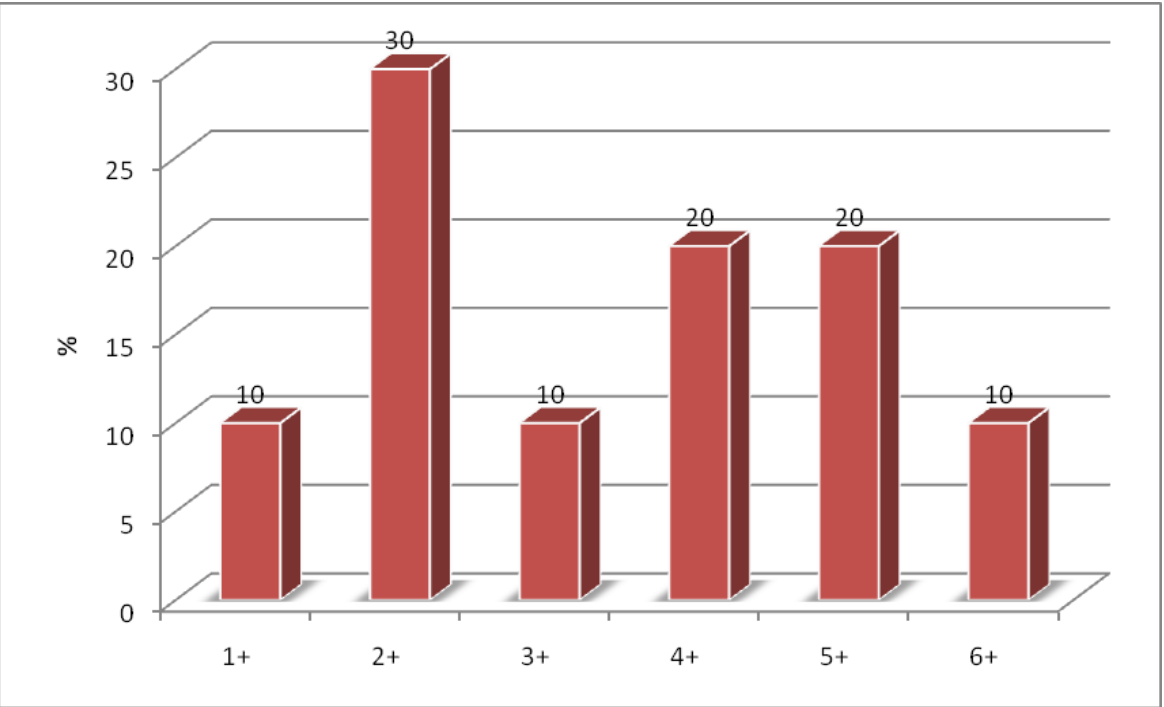

Fig. 4: Distribution of type of Bacillary Index in Multi bacillary

Table-4: Comparison of clinical diagnosis with histopathological diagnosis

\begin{tabular}{|c|c|c|c|c|c|c|c|c|c|c|c|c|c|}
\hline \multirow{3}{*}{$\begin{array}{l}\text { Clinical } \\
\text { diagnosis }\end{array}$} & \multirow{3}{*}{$\begin{array}{c}\text { No. of } \\
\text { patients }\end{array}$} & \multicolumn{12}{|c|}{ Histopathological examination } \\
\hline & & \multicolumn{2}{|c|}{ TT } & \multicolumn{2}{|c|}{ BT } & \multicolumn{2}{|c|}{ BB } & \multicolumn{2}{|c|}{$\mathbf{B L}$} & \multicolumn{2}{|c|}{ IL } & \multicolumn{2}{|c|}{ LL } \\
\hline & & No. & $\%$ & No. & $\%$ & No. & $\%$ & No. & $\%$ & No. & $\%$ & No. & $\%$ \\
\hline TT & 14 & 1 & 7.1 & 4 & 28.6 & 2 & 14.3 & 1 & 7.1 & 5 & 35.7 & 1 & 7.1 \\
\hline BT & 9 & 0 & 0.0 & 1 & 11.1 & 1 & 11.1 & 2 & 22.2 & 2 & 22.2 & 3 & 33.3 \\
\hline BB & 6 & 0 & 0.0 & 0 & 0.0 & 1 & 16.7 & 0 & 0.0 & 3 & 50.0 & 2 & 33.3 \\
\hline BL & 5 & 0 & 0.0 & 2 & 40.0 & 1 & 20.0 & 0 & 0.0 & 0 & 0.0 & 2 & 40.0 \\
\hline IL & 7 & 1 & 14.3 & 3 & 42.9 & 1 & 14.3 & 0 & 0.0 & 0 & 0.0 & 2 & 28.6 \\
\hline LL & 12 & 2 & 16.7 & 2 & 16.7 & 1 & 8.3 & 2 & 16.7 & 4 & 33.3 & 1 & 8.3 \\
\hline
\end{tabular}

BT was correlated in $11.1 \%$ of the cases in clinical and histopathological diagnosis. However, TT correlated in $7.1 \%$ (Table-4). 